$11-1-2004$

\title{
On Comparison of Hypothesis Tests in the Bayesian Framework without Loss Function
}

\author{
Vladimir Gercsik \\ Russian Academy of Science \\ Mark Kelbert \\ University of Wales-Swansea, United Kingdom, M.Kelbert@swansea.ac.uk
}

Follow this and additional works at: http:// digitalcommons.wayne.edu/jmasm

Part of the Applied Statistics Commons, Social and Behavioral Sciences Commons, and the Statistical Theory Commons

\section{Recommended Citation}

Gercsik, Vladimir and Kelbert, Mark (2004) "On Comparison of Hypothesis Tests in the Bayesian Framework without Loss Function," Journal of Modern Applied Statistical Methods: Vol. 3 : Iss. 2 , Article 12.

DOI: $10.22237 /$ jmasm/1099267920

Available at: http://digitalcommons.wayne.edu/jmasm/vol3/iss2/12

This Regular Article is brought to you for free and open access by the Open Access Journals at DigitalCommons@WayneState. It has been accepted for inclusion in Journal of Modern Applied Statistical Methods by an authorized editor of DigitalCommons@WayneState. 


\section{On Comparison of Hypothesis Tests in the Bayesian Framework without Loss Function}

\author{
Vladimir Gercsik \\ International Institute of Earthquake \\ Prediction Theory \& Mathematical Geophysics \\ Russian Academy of Science
}

\author{
Mark Kelbert \\ Department of Mathematics \\ University of Wales-Swansea \\ United Kingdom
}

The problem is how to compare the quality of different hypothesis tests in a Bayesian framework without introducing a loss function. Three different linear orders on the set of all possible hypothesis tests are studied. The most natural order estimates the Fisher information between indicators of event and decision.

Key words: Bayesian risk, Neyman-Pearson test, Fisher information, tests of independency

Introduction

It is well-known that no universal measure of a hypothesis test quality exists in statistics. In the Bayesian framework a linear ordering of tests is possible for a given loss function. It is said that a test is optimal if it minimizes the Bayesian risk.

However, the selection of a loss function is often somewhat arbitrary, and it is not always natural to measure the losses under different types of errors in the same units. For example, the cost of prevention measures in earthquake prediction is naturally expressed in money units. However, the losses from an earthquake including the psychological traumas, maiming and even the loss of human lives could be hardly expressed in money terms. Even if this expression is imposed, any estimation of these losses in money terms would depend a great deal on the variable economical and political situation. This loss function hardly looks as neutral and scientifically unbiased.

The subject of interest is in a situation when the loss function is unknown but the quality of any two hypothesis tests should be quantitatively compared. It turned out that all

Vladimir Gercsik is a Senior Researcher in geophysics and earthquake prediction. Mark Kelbert is a Reader in statistics. Email him at M.Kelbert@swansea.ac.uk. hypothesis tests could be linearly ordered at least in three different ways:

Let $p$ and $1-p$ be the Bayesian probabilities of random experiments $\omega \in \Omega$ with cumulative distribution functions $F_{1}$ and $F_{2}$, respectively. A test is defined by a function $\Phi(\omega)=1$ on the critical set $B$ and 0 on $B^{c}$. If $\Phi(\omega)=1$ then the alternative $F_{2}$ is accepted, and the hypothesis $F_{1}$ is accepted in the case $\Phi(\omega)=0$. Clearly, the problem is symmetric with respect to interchange of hypothesis $F_{1}$ and $F_{2}$ and a simultaneous interchange of $\Phi(\omega)$ and $1-\Phi(\omega)$.

The type I error is denoted (i.e., the probability to accept $F_{2}$ when $F_{1}$ is true) by $\alpha_{1}$, and the type II error (i.e., the probability to accept $F_{1}$ when $F_{2}$ is true) by $\alpha_{2}$. Considered are only unbiased tests (Barra, 1981), i.e., assume that $\alpha_{1}+\alpha_{2} \leq 1$. (If this condition is violated one could get an unbiased test by selecting $B^{C}$ instead of $B$ as a critical set.) Consider a random variable $X_{1}=0$ if $F_{1}$ is true, $X_{1}=1$ if $F_{2}$ is true, and call it an indicator of events. In a similar manner we define a random variable $X_{2}=0$ if a test accepts $F_{1}$, and $X_{2}=1$ if the test accepts $F_{2}$, called an indicator of decision. The joint distribution $\mathbf{P}\left(x_{1}, x_{2}\right)$ is defined by the relations $\alpha_{1}=\mathbf{P}\left(X_{2}=1 \mid X_{1}=0\right), \alpha_{2}=\mathbf{P}\left(X_{2}=0 \mid X_{1}=1\right)$, in particular, 


$$
\begin{aligned}
& \mathbf{P}\left(X_{2}=0, X_{1}=0\right)=p\left(1-\alpha_{1}\right), \\
& \mathbf{P}\left(X_{2}=1, X_{1}=0\right)=p \alpha_{1}, \\
& \mathbf{P}\left(X_{2}=0, X_{1}=1\right)=(1-p) \alpha_{2}, \\
& \mathbf{P}\left(X_{2}=1, X_{1}=1\right)=(1-p)\left(1-\alpha_{2}\right) .
\end{aligned}
$$

The marginal one-dimensional probabilities take the form:

$$
\begin{aligned}
& \mathbf{P}\left(X_{1}=0\right)=p, \mathbf{P}\left(X_{1}=1\right)=(1-p), \\
& \mathbf{P}\left(X_{2}=0\right)=p\left(1-\alpha_{1}\right)+\alpha_{2}(1-p), \\
& \mathbf{P}\left(X_{2}=1\right)=p \alpha_{1}+(1-p)\left(1-\alpha_{2}\right) .
\end{aligned}
$$

Clearly, the worst possible unbiased test is determined by the condition that indicator of event $X_{1}$ and indicator of decision $X_{2}$ are independent. On the other extreme, an ideal test (normally, it does not exist) is one that provides the correct solution without any errors. Generically, the quality of a test is measured by some non-negative function of $X_{1}$ and $X_{2}=\Phi(\omega)$ which takes the value 0 iff $X_{1}, X_{2}$ are independent, and the value 1 iff $X_{1}=X_{2}$.

Measuring the quality of a test

Any of the following well-known Rachev (1991) functions used in tests of independency is acceptable as a measure of the quality of a test.

$$
\begin{aligned}
& \beta_{1}(\boldsymbol{\Phi})=\sup _{x_{1}, x_{2}}\left|\mathbf{P}\left(x_{1}, x_{2}\right)-\mathbf{P}\left(x_{1}\right) \mathbf{P}\left(x_{2}\right)\right|, \\
& \beta_{2}(\Phi)=\mathbf{E}_{x_{1}}\left[\sup _{x_{2}}\left|\mathbf{P}\left(x_{2} \mid x_{1}\right)-\mathbf{P}\left(x_{2}\right)\right|\right]
\end{aligned}
$$

( $\mathbf{E}_{x_{1}}$ stands for the expectation with respect of distribution of $X_{1}$ ). It is easy to check that

$$
\beta_{1}(\Phi)=\beta_{2}(\Phi)=p(1-p)\left(1-\alpha_{1}-\alpha_{2}\right) .
$$

The quality of a test is measured by $\beta=\left(1-\alpha_{1}-\alpha_{2}\right)$. This is quite popular in practice as the Bayesian risk $\mathbf{R}=\mathbf{E}[w]=\alpha_{1}+\alpha_{2}$ appears for the simplest loss function $w\left(x_{1}, x_{2}\right)$ : $w(0,0)=w(1,1)=0, w(0,1)=w(1,0)=1$.
Another possibility to test the independence is to consider the maximal correlation coefficient

$$
\rho(\boldsymbol{\Phi})=\sup \mathbf{E}\left[\phi_{1}\left(X_{1}\right) \phi_{2}\left(X_{2}\right)\right]
$$

where sup is taken over the set of functions $\phi_{1}$, $\phi_{2}$ such that $\mathbf{E}\left[\phi_{i}\left(X_{i}\right)\right]=0, \sigma^{2}\left[\phi_{i}\left(X_{i}\right)\right]=\mathbf{E}\left[\phi_{i}\right.$ $\left.\left(X_{i}\right)^{2}\right]=1, i=1,2$. Clearly, $\rho(\Phi)=0$ iff $X_{1}$ and $X_{2}$ are independent. As function $\phi_{\mathrm{i}}(\mathrm{x}), \mathrm{i}=1,2$ could take only two values $\phi_{\mathrm{i}}(0)$ and $\phi_{\mathrm{i}}(1)$, and these values are defined in a unique way by the conditions imposed, the following relation holds

$$
\rho(\mathbf{\Phi})=\mathbf{E}\left[\frac{\left(X_{1}-\mathbf{E}\left(X_{1}\right)\right)}{\sigma\left(X_{1}\right)} \frac{\left(X_{2}-\mathbf{E}\left(X_{2}\right)\right)}{\sigma\left(X_{2}\right)}\right]
$$

A straightforward computation yields:

$$
\rho(\boldsymbol{\Phi})=\beta \sqrt{\frac{p(1-p)}{P(1-P)}}
$$

where

$$
\mathbf{P}=\mathbf{P}\left(X_{2}=0\right)=\alpha_{2}(1-p)+\left(1-\alpha_{1}\right) p=\alpha_{2}+\beta p .
$$

The correlation coefficient $\rho(\Phi)$ is non-negative for any unbiased test; it equals to 1 when $X_{1}=$ $X_{2}$.

Perhaps, the most interesting way to measure the quality of a test is to consider an information $I(\Phi)$ in the indicator of solution $X_{2}=\Phi$ about the indicator of event $X_{1}$. To formalize this idea consider

$$
\begin{aligned}
& I(\boldsymbol{\Phi})= \\
& \sum_{x_{1}=0,1} \sum_{x_{2}=0,1} \mathbf{P}\left(x_{1}, x_{2}\right) \log _{2} \frac{\mathbf{P}\left(x_{1}, x_{2}\right)}{\mathbf{P}\left(x_{1}\right) \mathbf{P}\left(x_{2}\right)}
\end{aligned}
$$

which equals to $I(\Phi)=S\left(X_{1}\right)-\mathbf{E}\left[S\left(X_{1} \mid X_{2}\right)\right]$.

$\mathrm{S}\left(X_{1}\right)$ stands for the Fisher information of the prior distribution $\mathbf{P}\left(x_{1}\right), \quad \mathrm{S}\left(X_{1}\right)=p$ $\log _{2} p+(1-p) \log _{2}(1-p)$. Intuitively, it means that in a random trial with $n$ outcomes where hypothesis $F_{1}$ and $F_{2}$ appear with probabilities $p$ and $1-p$, respectively, there are $\approx 2^{\mathrm{nS}}$ quite 
probable outcomes, and the rest could be neglected as $n \rightarrow \infty$. Next, $\mathrm{S}\left(X_{1} \mid X_{2}\right)$ is the conditional entropy of the conditional distribution $\mathbf{P}\left(\mathrm{x}_{1} \mid \mathrm{x}_{2}\right)$ under condition that the decision $\mathrm{x}_{2}$ is taken.

Therefore, the information $I(\Phi)$ equals to the mean reduction of uncertainty obtained by the using of the test $\boldsymbol{\Phi}$. Clearly, for an ideal test without any errors $\mathrm{I}(\Phi)$ takes its maximal value $\mathrm{S}\left(X_{1}\right)$, and $I(\Phi)=0$ iff $X_{1}$ and $X_{2}$ are independent.

It is interesting to note that a sequence of random events $X_{1}^{(n)}$ and decisions $X_{2}{ }^{(n)}$ $=1,2, \ldots$ can be treated as a message transmission over a channel without noise. In this interpretation the observation $\mathrm{x}_{1}{ }^{(\mathrm{n})}(\omega)$ could be treated as the coding of the random outcome $F_{\mathrm{i}}$, $i=1,2$, and the decision $\mathrm{x}_{2}{ }^{(\mathrm{n})}(\omega)$ as its decoding. The maximization of $I(\Phi)$ means that the optimal decoding is applied.

Now following is proved:

Lemma 1. Fix the Type I error probability $\alpha_{1}$. Then, the Neyman-Pearson test $\Phi^{*}$ minimizing the Type II error probability $\alpha_{2}$, maximizes also the information $I\left(\Phi^{*}\right)$ among all unbiased tests.

yields

Proof. A straightforward computation

$$
\begin{aligned}
& \frac{\partial I}{\partial \alpha_{2}}= \\
& (1-p)\left[\log _{2}\left(1-p+\frac{\alpha_{1} p}{1-\alpha_{2}}\right)-\log _{2}\left(1-p+\frac{\left(1-\alpha_{1}\right) p}{\alpha_{2}}\right)\right]
\end{aligned}
$$

This derivative is non-positive for an unbiased test with $\alpha_{1}+\alpha_{2} \leq 1$. Hence, the information $I(\Phi)$ is maximal for a minimal possible value of $\alpha_{2}$, i.e. for the test $\Phi^{*}$. A symmetric statement with interchanging of $\alpha_{1}$ and $\alpha_{2}$ is also true. $\bullet$

The same property holds also for $\beta(\Phi)$ and $\rho(\Phi)$; this is immediate for $\beta(\Phi)$, and follows from the equality

$$
\begin{aligned}
& \frac{\partial \rho}{\partial \alpha_{2}}= \\
& -\frac{\alpha_{1} P+\left(1-\alpha_{1}\right)(1-P)}{P(1-P)} \sqrt{\frac{p(1-p)}{P(1-P)}} \leq 0
\end{aligned}
$$

in the case of $\rho(\Phi)$.

Next, observe that the Fisher information $I(\Phi)$ is a convex function of $\alpha_{1}$ and $\alpha_{2}$ for all $0<\alpha_{1}<1,0<\alpha_{2}<1,0<p<1$. The prove this, it suffices to compute

$$
\begin{gathered}
\frac{\partial^{2} I}{\partial \alpha_{2}^{2}}=p\left[\frac{P(1-P)-p \alpha_{1}\left(1-\alpha_{1}\right)}{\alpha_{1}\left(1-\alpha_{1}\right) P(1-P)}\right], \\
\frac{\partial^{2} I}{\partial \alpha_{1}^{2}}=(1-p)\left[\frac{P(1-P)-p \alpha_{2}\left(1-\alpha_{2}\right)}{\alpha_{2}\left(1-\alpha_{2}\right) P(1-P)}\right], \\
\frac{\partial^{2} I}{\partial \alpha_{1} \partial \alpha_{2}}=\frac{p(1-p)}{P(1-P)}, \\
\operatorname{det}\left\|\frac{\partial^{2} I}{\partial \alpha_{i} \partial \alpha_{j}}\right\|= \\
\frac{p(1-p)\left(1-\alpha_{1}-\alpha_{2}\right)^{2}}{P(1-P) \alpha_{1}\left(1-\alpha_{1}\right) \alpha_{2}\left(1-\alpha_{2}\right)}>0 .
\end{gathered}
$$

This convexity property implies that a randomized test $\overline{\boldsymbol{\Phi}}=\int_{\boldsymbol{\Phi} \in A} \boldsymbol{\Phi} \mathbf{P}(d \boldsymbol{\Phi})$ where $\mathbf{P}$ $(\mathrm{d} \Phi)$ is a probability measure on a suitable set A, $\mathbf{P}(\mathrm{A})=1$, can not be optimal in the sense of Fisher information. Indeed, Jensen's inequality yields

$$
\begin{gathered}
I(\overline{\mathbf{\Phi}})=I\left(\int_{\boldsymbol{\Phi} \in A} \boldsymbol{\Phi} \mathbf{P}(d \boldsymbol{\Phi})\right) \leq \\
\int_{\boldsymbol{\Phi} \in A} I(\boldsymbol{\Phi}) \mathbf{P}(d \boldsymbol{\Phi}) \leq \sup _{\Phi \in A} I(\boldsymbol{\Phi})
\end{gathered}
$$

Hence, there always exists a non-randomized test $\Phi^{\prime}$ such that $I(\overline{\boldsymbol{\Phi}}) \leq I\left(\boldsymbol{\Phi}^{\prime}\right)$. Clearly, a similar statement holds for $\beta(\Phi)$ as well. However, it is not true generically for $\rho(\Phi)$. 
Fig.1 demonstrates this presenting the surface $\rho\left(\alpha_{1}, \alpha_{2}\right)$ for $p=1 / 3$. Fig.2 presents the surface $I\left(\alpha_{1}, \alpha_{2}\right)$ for $p=1 / 3$. Each of the characteristics $\beta(\Phi), \rho(\Phi)$ and $\mathrm{I}(\Phi)$ divides the set of all tests into equivalence classes with the same value of this characteristic inside the class, and defines a linear order between different equivalence classes.

\section{Numerical Examples}

Figure 3 presents the results of computation of optimal tests with respect of three criteria as above. As hypothesis $F_{1}$ and $F_{2}$, select the normal distributions with pdf's

$$
\begin{aligned}
& f_{1}(x)=\frac{1}{\sqrt{2 \pi}} \exp \left(-\frac{(x+0.5)^{2}}{2}\right), \\
& f_{2}(x)=\frac{1}{\sqrt{2 \pi}} \exp \left(-\frac{(x-0.5)^{2}}{2}\right),
\end{aligned}
$$

respectively. The prior probability of appearing $F_{1}$ is $p=1 / 3$. Fig.3 presents the type II error $\alpha_{2}=g\left(\alpha_{1}\right)$ for Neyman-Pearson's problem in the cases $\sigma^{2}=0.2, \sigma^{2}=0.6$ and $\sigma^{2}=1$. Each of these curves represents all the tests of the form

$$
\boldsymbol{\Phi}(x)= \begin{cases}1, & \text { if } f_{2}(x) \geq \lambda f_{1}(x) \\ 0, & \text { if } f_{2}(x)<\lambda f_{1}(x)\end{cases}
$$

with different $\lambda$ which are optimal for NeymanPearson problem, i.e., minimizes $\alpha_{2}$ for a given value of $\alpha_{1}$. These curves of errors serve as a boundary of a convex domain of errors for all possible tests. The points are indicated $\left(\alpha_{1}, \alpha_{2}\right)$ on the boundary where each of three characteristics of quality as above achieves its maximum. Clearly, the position of maximum is distinct in all three cases.

\section{Checking Hypothesis of Uselessness of a Test}

It is desirable to use empirical data for checking the uselessness of a test $\Phi$. In the case of independence of the indicator of event and the indicator of decision any of three characteristics of quality as above equals 0 . To reject the hypothesis of useless of test $\Phi$ with some level of confidence it is sufficient to demonstrate that $\alpha_{1}+\alpha_{2}<1$ with this level of confidence.

Consider a series of $n$ independent trials where the number $M$ of appearance of distribution $F_{2}$ equals $m$, the number $L$ of selection of distribution $F_{2}$ by the test $\Phi$ equals $l$, and the number of correct choices $K$ of $F_{2}$ by the test $\Phi$ equals $k$. The hypothesis of uselessness is formalized in the following form of $\mathrm{H}_{0}$ : $\alpha_{1}+\alpha_{2}=1$. If $H_{0}$ is true, then (with unknown probability $p$ )

$$
\begin{aligned}
& \mathbf{P}\left(X_{1}=0\right)=p, \\
& \mathbf{P}\left(X_{2}=0\right)=\alpha_{2}(1-p)+\left(1-\alpha_{1}\right) p \equiv \alpha_{2}
\end{aligned}
$$

and $X_{1}$ and $X_{2}$ are independent.

Probabilities $\mathbf{P}\left(X_{2}=0, X_{1}=0\right), \mathbf{P}\left(X_{2}=1\right.$, $\left.X_{1}=0\right), \mathbf{P}\left(X_{2}=0, X_{1}=1\right)$ and $\mathbf{P}\left(X_{2}=1, X_{1}=1\right)$ are estimated by the empirical frequencies $(n-m-1+k) / n, \quad(l-k) / n, \quad(m-k) / n \quad$ and $k / n$, respectively. Hence, the estimates of conditional probabilities $\alpha_{1}=\mathbf{P}\left(X_{2}=1 \mid X_{1}=0\right)$ and $\alpha_{2}=\mathbf{P}\left(X_{2}=0 \mid X_{1}=1\right)$ looks like $(\mathrm{l}-k) /(n-m)$ and $(m-k) / m$, respectively.

Clearly, the inequality $k>m l / n$ corresponds to the alternative $H_{1}: \alpha_{1}+\alpha_{2}<1$. It means that the critical sets have the form $\left\{K>K^{*}\right\}$ for given values $M=m$ and $L=1$.

If hypothesis $H_{0}$ is true then the independence of $X_{1}$ and $X_{2}$ and independence of different trials imply an explicit expression for test size

$$
\begin{aligned}
& \mathbf{P}\left(K>K^{*}, M=m, L=l\right)= \\
& \left(\begin{array}{c}
n \\
m
\end{array}\right) p^{m}(1-p)^{n-m} \times \\
& \min \{m, l\} \\
& \sum^{k}\left(\begin{array}{c}
m \\
k
\end{array}\right) q^{k}(1-q)^{m-k} \\
& k=K^{*} \\
& \left(\begin{array}{c}
n-m \\
l-k
\end{array}\right) q^{l-k}(1-q)^{n-m-l+k} \\
& =\left(\begin{array}{c}
n \\
m
\end{array}\right) p^{m}(1-p)^{n-m} q^{l}(1-q)^{n-l} \\
& \quad \min \{m, l\} \\
& \sum \\
& k=K^{*}\left(\begin{array}{c}
n-m \\
l-k
\end{array}\right)\left(\begin{array}{c}
m \\
k
\end{array}\right)
\end{aligned}
$$


Figure 1. Dependence of correlation coefficient on errors.

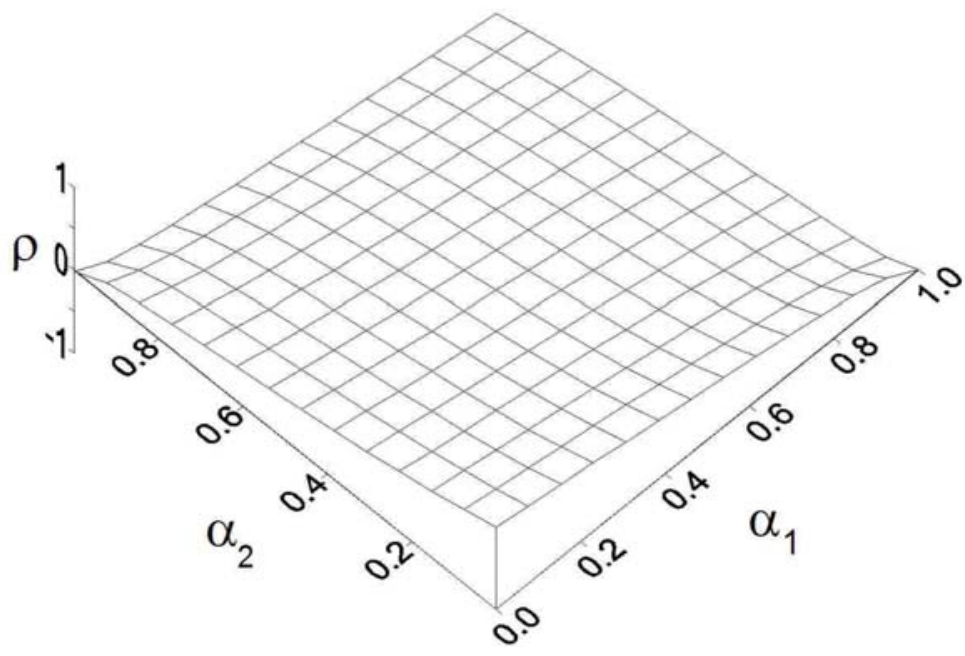

Figure 2. Dependence of Fisher information coefficient on errors.

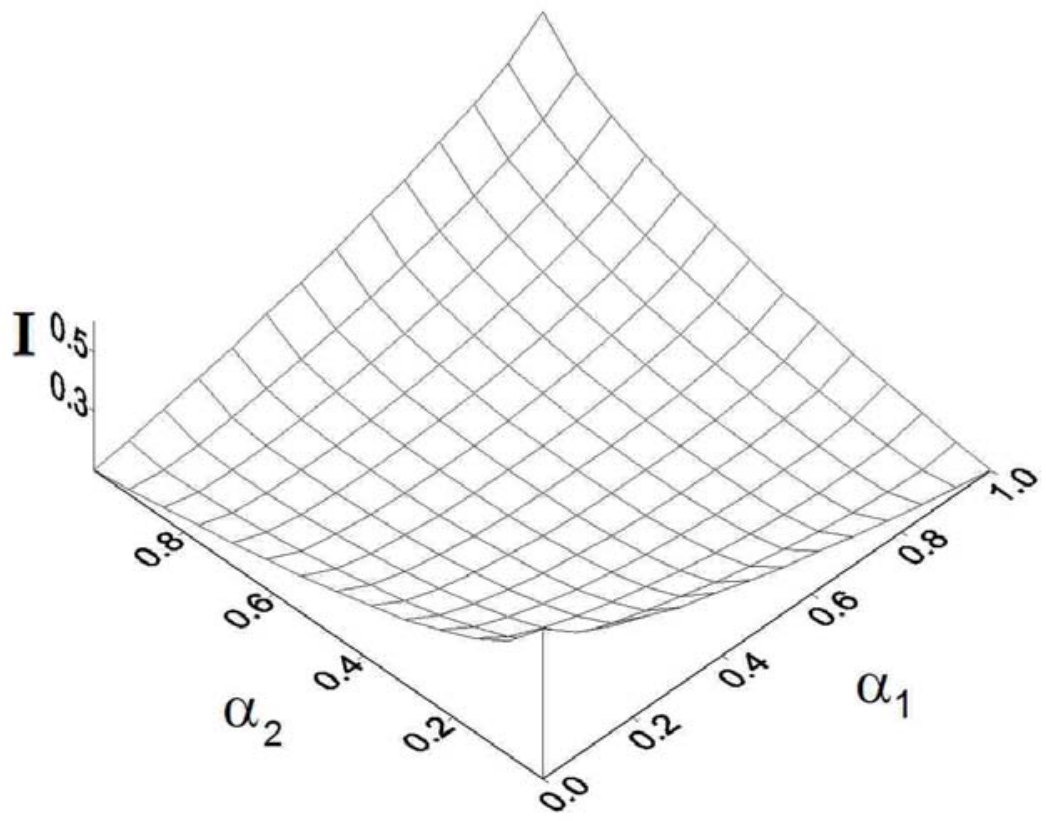


Figure 3. Location of maximum points for different characteristics of test efficiency.

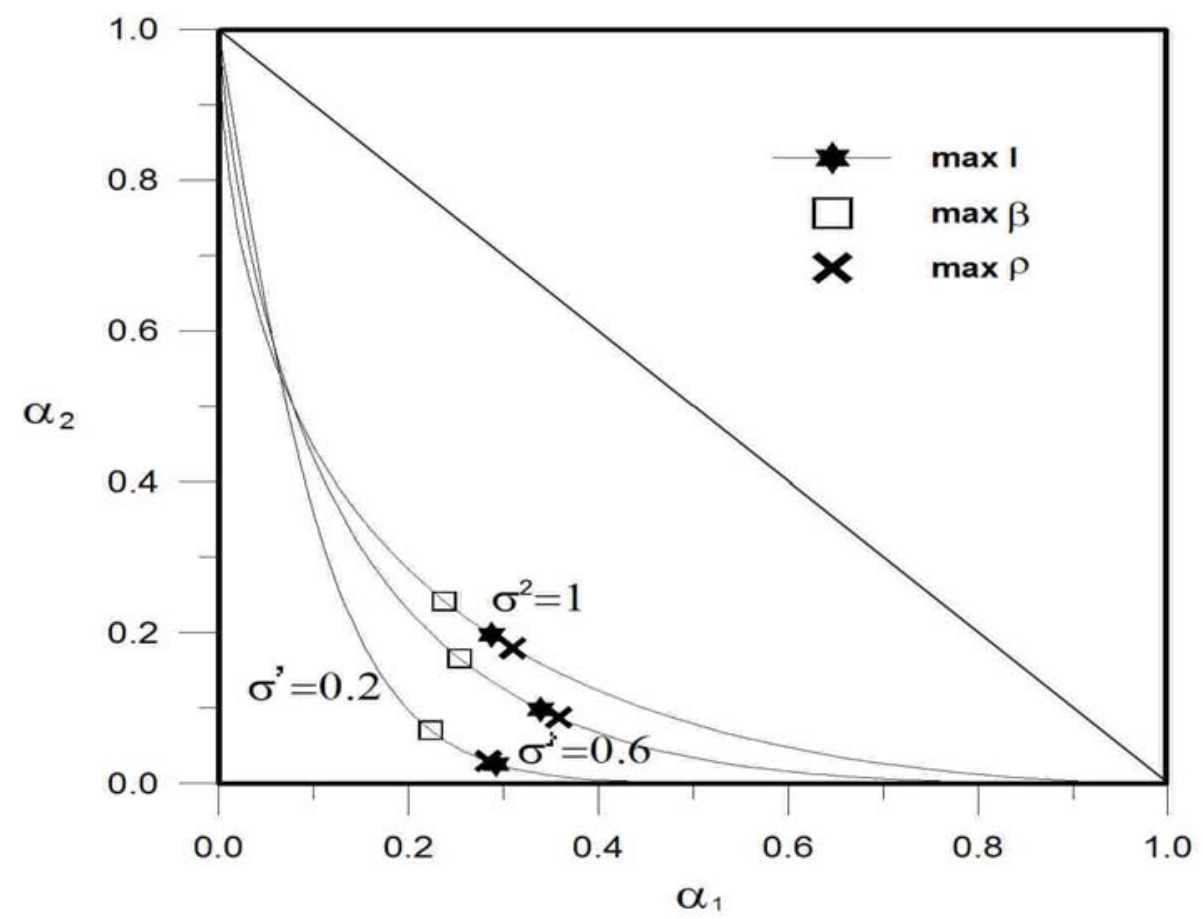

Figure 4. Dependence of maxima locations for different characteristics of test efficiency on variance.

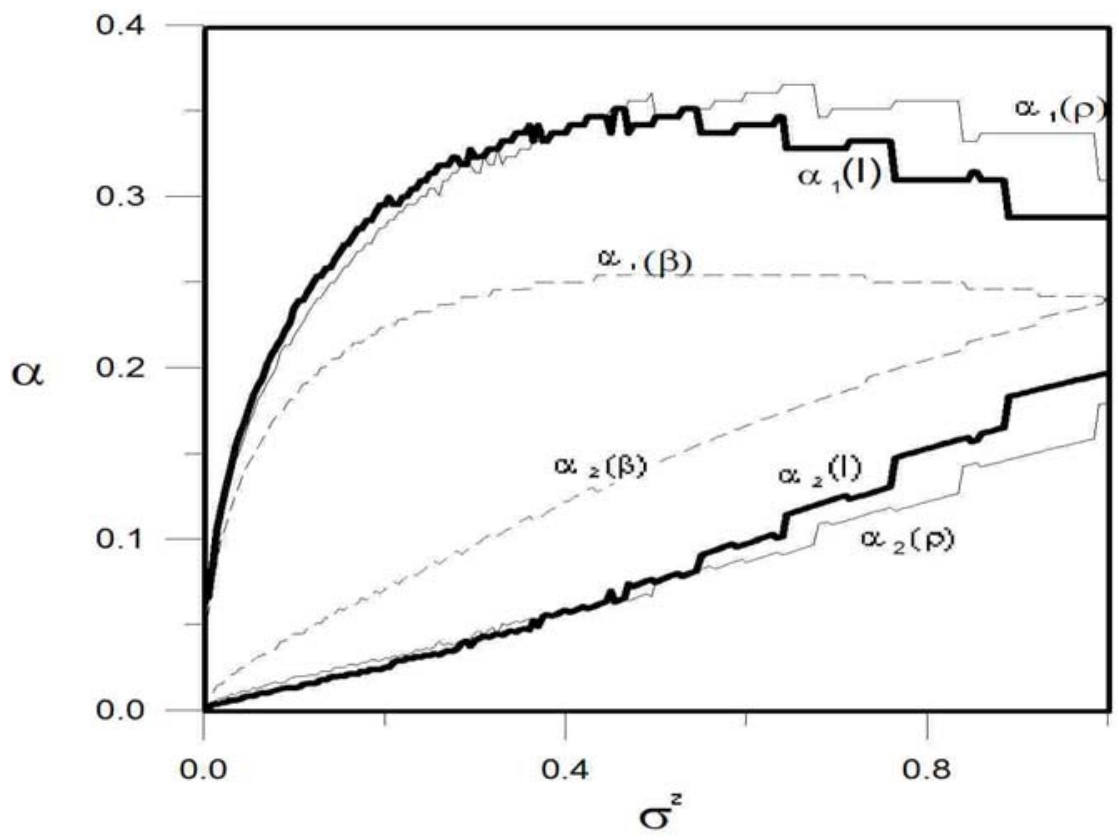

Fig. 4 presents the dependence of locations of maximum points $\alpha_{i}(\beta), \alpha_{i}(\rho)$ and $\alpha_{i}(I), i=1,2$, for three characteristics of quality as above as functions of $\sigma^{2}$. Observe that the Type II error tends to 0 as $\sigma^{2} \rightarrow 0$, as the PDF $f_{2}$ tends to a delta-function located at the point $x=0.5$. 
Here $q=\alpha_{2}$. Using the equalities

$$
\begin{aligned}
& \mathbf{P}(M=m)=\left(\begin{array}{c}
n \\
m
\end{array}\right) p^{m}(1-p)^{n-m}, \\
& \mathbf{P}(L=l)=\left(\begin{array}{c}
n \\
l
\end{array}\right) q^{l}(1-q)^{n-l},
\end{aligned}
$$

obtain

$$
\mathbf{P}\left(K \geq K^{*} \mid M=m, L=l\right)=\sum_{k=K^{*}}^{\min \{m, l\}} \frac{\left(\begin{array}{l}
m \\
k
\end{array}\right)\left(\begin{array}{c}
n-m \\
l-k
\end{array}\right)}{\left(\begin{array}{l}
n \\
l
\end{array}\right)}
$$

This conditional probability represents the level of confidence for the critical region $\left\{K>K^{*}\right\}$ for given values $M=m$ and $L=1$.

\section{References}

Barra J. R. (1981). Mathematical basis of statistics. New York: Academic Press.

Rachev S. (1991). Probability metrics and the stability of stochastic models. Chichester: John Wiley. 